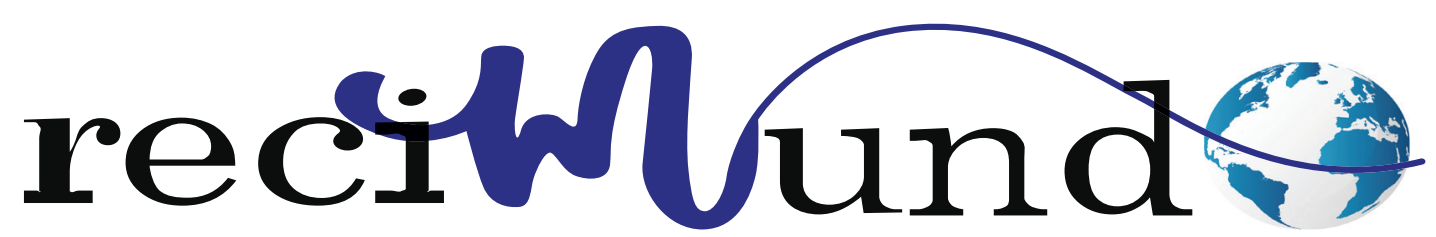

Revista Científica Mundo de la Investigación y el Conocimiento

DOI: 10.26820/recimundo/5.(1).enero.2021.4-19

URL: http://recimundo.com/index.php/es/article/view/978

EDITORIAL: Saberes del Conocimiento

REVISTA: RECIMUNDO

ISSN: 2588-073X

TIPO DE INVESTIGACIÓN: Artículo de Cientifico

CÓDIGO UNESCO: 1203 Ciencia de los Ordenadores

PAGINAS: 4-19

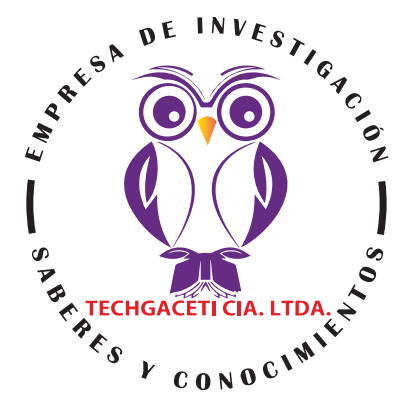

\title{
Estudio del uso de técnicas de inteligencia artificial aplicadas para análisis de suelos para el sector agrícola
}

Study of the use of artificial intelligence techniques applied to the analysis of soils for the agricultural sector

Estudo do uso de técnicas de inteligência artificial aplicadas à análise de solos para o setor agrícola

Juan Sebastian Bonilla Segovia'; Francisco Andrés Dávila Rojas²; Manuel William Villa Quishpe ${ }^{3}$

RECIBIDO: 28/11/2020 ACEPTADO: 06/12/2020 PUBLICADO: 31/01/2021

1. Universidad Técnica Cotopaxi, Latacunga, Ecuador; juan.bonilla4105@utc.edu.ec; iD https://orcid.org/0000-0002-74128176

2. Universidad Técnica Cotopaxi, Latacunga, Ecuador; francisco.davila2@utc.edu.ec; (iD https://orcid.org/0000-0002-45042496

3. Magister en Interconectividad de Redes; Diploma Superior en Comercio Exterior; Licenciado en Sistemas Computacionales; Ingeniero en Sistemas e Informática; Universidad Técnica Cotopaxi, Latacunga; Ecuador; manuel.villa@utc.edu.ec; (iD https://orcid.org/0000-0001-5827-8337

CORRESPONDENCIA

Juan Sebastian Bonilla Segovia

juan.bonilla4105@utc.edu.ec

Latacunga, Ecuador

○ RECIMUNDO; Editorial Saberes del Conocimiento, 2021 


\section{RESUMEN}

La aplicación de la inteligencia artificial (IA) ha sido evidente en el sector agrícola. Hoy en día, la agricultura se enfrenta a numerosos desafíos para maximizar su rendimiento, incluidos tratamientos inadecuados del suelo, infestación de enfermedades, plagas, entre otros, por lo que la necesidad del manejo de big data se ha vuelto un requisito fundamental en este sector para incrementar el conocimiento entre los agricultores y la tecnología. El concepto principal de IA en la agricultura es su flexibilidad, alto rendimiento, precisión y rentabilidad. La automatización de la agricultura es la principal preocupación y el tema emergente de todos los países, ya que, los métodos tradicionales utilizados por los agricultores ya no son suficientes para satisfacer la creciente demanda de aumento de la población. En relación con esto, se introdujeron nuevos métodos automatizados que satisfacen las necesidades alimentarias y proporcionan grandes oportunidades de empleo, trayendo una revolución agrícola en este sector. El uso de inteligencia artificial y de las nuevas tecnologías ha protegido el cultivo a través del rendimiento de varios factores como los cambios climáticos, el crecimiento de la población, los problemas de empleo y la seguridad alimentaria, brindando beneficios en el uso adecuado del agua, pesticidas, herbicidas, así como también, ayudando en el uso eficiente de la mano de obra, elevando la productividad, mejorando la calidad y manteniendo la fertilidad del suelo. Este artículo presenta una revisión de las aplicaciones de la IA en el suelo, manejo de cultivos, manejo de malezas y enfermedades por administración. Se presta especial atención a la fuerza y limitaciones de la aplicación y la forma de utilizar expertos sistemas para una mayor productividad.

Palabras clave: Inteligencia artificial, agricultura, manejo del suelo, manejo de cultivos, rendimiento.

\section{ABSTRACT}

The application of artificial intelligence (Al) has been evident in the agricultural sector. Today, agriculture faces numerous challenges to maximize its yield, including inadequate soil treatments, disease infestation, pests, among others, so the need to manage big data has become a fundamental requirement in this sector to increase knowledge among farmers and technology. The main concept of Al in agriculture is its flexibility, high performance, precision and cost effectiveness. The automation of agriculture is the main concern and the emerging issue of all countries, since the traditional methods used by farmers are no longer sufficient to meet the growing demand of increasing population. In relation to this, new automated methods were introduced that meet food needs and provide great employment opportunities, bringing an agricultural revolution in this sector. The use of artificial intelligence and new technologies has protected the crop through the performance of various factors such as climate changes, population growth, employment problems and food security, providing benefits in the proper use of water, pesticides, herbicides, as well as helping in the efficient use of labor, raising productivity, improving quality and maintaining soil fertility. This article presents a review of Al applications in soil, crop management, weed management, and disease management. Particular attention is paid to the strength and limitations of the application and how to use expert systems for increased productivity.

Keywords: Artificial intelligence, agriculture, soil management. crop management, yield.

\section{RESUMO}

A aplicação da inteligência artificial (IA) tem sido evidente no setor agrícola. Hoje, a agricultura enfrenta inúmeros desafios para maximizar seu rendimento, incluindo tratamentos inadequados do solo, infestação de doenças, pragas, entre outros, de modo que a necessidade de gerenciar grandes dados tornou-se um requisito fundamental neste setor para aumentar o conhecimento entre os agricultores e a tecnologia. O principal conceito de IA na agricultura é sua flexibilidade, alto desempenho, precisão e custo-benefício. A automação da agricultura é a principal preocupação e a questão emergente de todos os países, já que os métodos tradicionais utilizados pelos agricultores não são mais suficientes para atender à crescente demanda do aumento da população. Em relação a isto, foram introduzidos novos métodos automatizados que atendem às necessidades alimentares e oferecem grandes oportunidades de emprego, trazendo uma revolução agrícola neste setor. O uso de inteligência artificial e novas tecnologias tem protegido a cultura através do desempenho de vários fatores como mudanças climáticas, crescimento populacional, problemas de emprego e segurança alimentar, proporcionando benefícios no uso adequado da água, pesticidas, herbicidas, além de ajudar no uso eficiente da mão-de-obra, aumentando a produtividade, melhorando a qualidade e mantendo a fertilidade do solo. Este artigo apresenta uma revisão das aplicações da IA no solo, no manejo de culturas, no manejo de ervas daninhas e no manejo de doenças. É dada especial atenção à força e limitações da aplicação e como usar sistemas especializados para aumentar a produtividade.

Palavras-chave: Inteligência artificial, agricultura, manejo do solo. manejo de culturas, rendimento. 


\section{Introducción}

La agricultura es la base de la sostenibilidad de cualquier economía. Desempeña un papel clave en el crecimiento económico a largo plazo, sin embargo, puede variar según los países. En el pasado, las actividades agrícolas se limitaban a la alimentación y producción de cultivos. Pero en las últimas dos décadas, ha evolucionado al procesamiento, producción, comercialización y distribución de cultivos y productos pecuarios. Actualmente, las actividades agrícolas sirven como fuente básica de sustento, mejorando el PIB (Producto Interno Bruto), siendo una fuente de comercio nacional, lo que reduce el desempleo, proporciona materias primas para la producción en otras industrias, y en general incrementa en el desarrollo de la economía.

Con la geometría global el aumento de la población se vuelve imperativo y las prácticas agrícolas se revisan con el objetivo de ofrecer enfoques innovadores para sostener y mejorar las actividades del sector. La introducción de la IA en la agricultura "será posible gracias a todos los avances tecnológicos, incluidos el análisis de macro datos, robótica, internet, la disponibilidad de sensores baratos y cámaras, tecnología de drones e incluso internet a gran escala con cobertura en campos geográficamente dispersos"(Zibah, 2015).

En tal sentido, analizando el suelo las fuentes de datos de gestión como la temperatura, el clima, la humedad y el rendimiento histórico de los cultivos; los sistemas de IA podrán proporcionar información predictiva sobre qué cultivo plantar en un año dado y cuando son las fechas óptimas para sembrar y cosechar en un área específica, mejorando así la producción de los cultivos y disminuyendo el uso de agua, fertilizantes y pesticidas.

Estas aplicaciones han dado múltiples ventajas a los productores de tierras, tomando en cuenta que esta labor representa un pilar fundamental en la economía de muchos países. SofOs, (2017) "razón por la cual con la llegada de las nuevas tecnologías en la agricultura se disminuyó en gran parte las cargas de trabajo pesado para los agricultores y estos aumentaron su producción".

La presión sobre el sector agrícola aumentará con la expansión continua de la población humana y, por lo tanto, la tecnología agrícola y la agricultura de precisión han ganado mucha importancia en el mundo actual. "Esto también se denomina agricultura digital, lo que significa el uso de sistemas informáticos de alta tecnología para calcular diferentes parámetros, como detección de malezas, predicción de cultivos, detección de rendimiento, calidad de cultivos y muchas técnicas de aprendizaje automático" (Liakos \& Busato, 2018)

Mediante el desarrollo del proceso investigativo, se hace mención a los diferentes usos de las técnicas de inteligencia artificial que se emplean en el sector agrícola a través de una revisión de la literatura, en aras de brindar información de interés para futuras investigaciones y para lectores que desean documentarse acerca de este tema.

\section{Metodología}

Para la realización de este trabajo se debe seguir un lineamiento en la cual permita poder desarrollar metodológicamente el análisis de las técnicas de IA que se aplican en los suelos del sector agrícola. Para lograr este fin, se emplean técnicas que permitan obtener información tales como encuestas, entrevistas y observación. En este caso la técnica de investigación utilizada fue el de las encuestas al personal que labora en los invernaderos.

\section{Tipo de investigación}

Existen diversos tipos de investigación aplicables a la investigación planteada. "Se 
tiene una investigación de campo ya que consiste en la recolección de datos directamente de la realidad donde ocurren los hechos" Palella \& Martins, (2010). Este trabajo tiene este tipo de investigación debido a que los datos e información son sacados de las personas encuestadas principales es decir de los empleados de los invernaderos y su experiencia con la tecnología de la información.

Dentro del tipo de investigación de campo se encuentra el nivel exploratorio. "Permite focalizar el tópico de interés, y/o delimitar futuros temas de investigación" Palella \& Martins, (2010). El poder analizar el uso de técnicas de inteligencia artificial para el análisis de suelos para el sector agrícola, puede permitir formar nuevas investigaciones a futuro para solventar diversas situaciones.

También se considera una investigación de tipo descriptiva, ya que, depende de la interpretación porque revela los rasgos interpretativos del investigador y la información sesgada, personal, de los sujetos informantes, además de los sesgos inherentes a la utilización de instrumentos, técnicas y métodos (Rojas, 2010). La utilización de técnicas de investigación permite obtener datos de primera mano de los trabajadores de los invernaderos, haciendo que sea una información subjetiva que dependerá de la apreciación del autor de este trabajo.

\section{Técnicas o instrumento de la investiga- ción}

Las técnicas utilizadas para la realización de este proyecto es la encuesta. "La encuesta es una técnica destinada a obtener datos de varias personas cuyas opiniones interesan al investigador. Se utiliza un listado de preguntas escritas que se entregan a los sujetos quienes, en forma anónima las responden por escrito" (Palella \& Martins, 2010).

\section{Base de Datos}

La base de datos utilizada fue la PhPM y Admin. "Esta base de datos es una herramienta escrita en PHP con la intención de manejar la administración a través de páginas web, utilizando Internet" (INC Web Hosting, 2020). Con esta herramienta se crea y eliminan bases de datos, se alteran tablas, se borran, editan y añaden campos, se administran claves en campos, se administran privilegios, se exportan datos en varios formatos, entre otros.

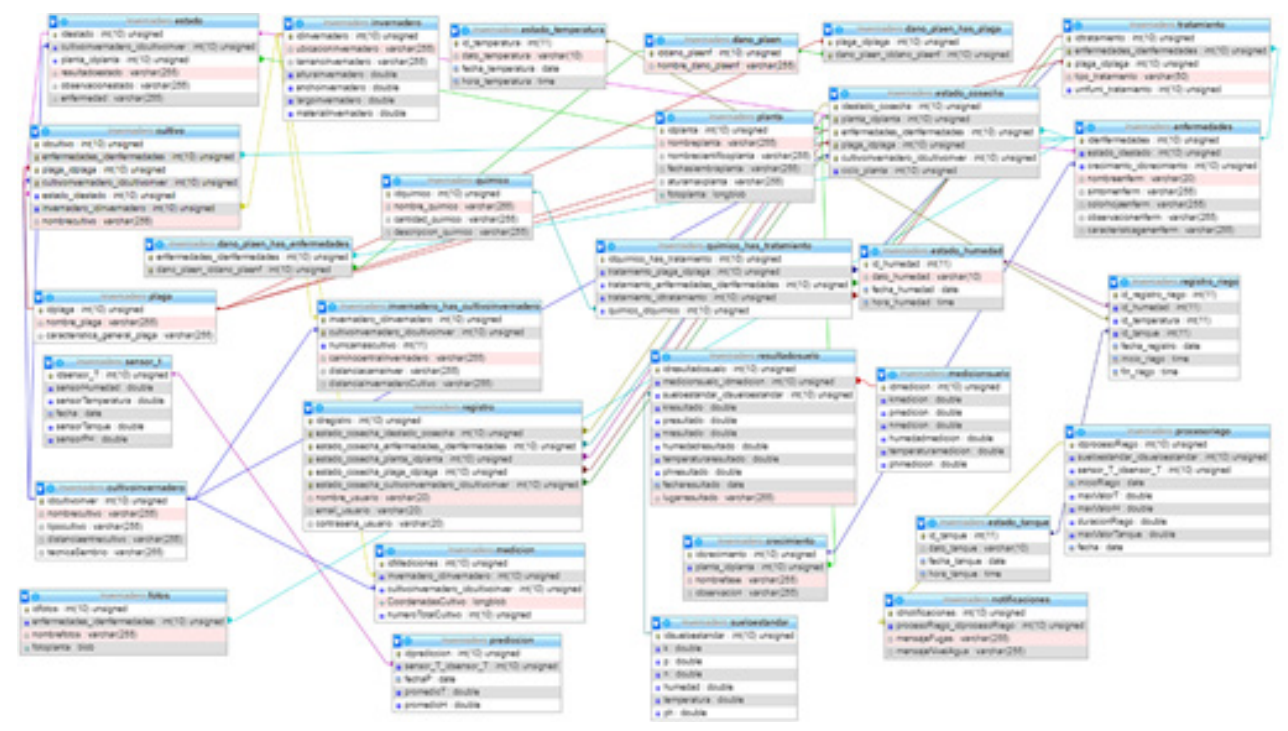

Figura 1. Base de Datos

Fuente: Grupo Investigación 
Ha servido como una herramienta fundamental en el sector de la agricultura, almacenando datos y tomando como referencia las características del cultivo de acuerdo a su crecimiento, desarrollo, medición y demás datos. El desarrollo del sistema de información se realizó por medio del lenguaje de programación Android y la metodología móvil MOBILE-D.

En este sentido, Android nativo, por estar basado en Java y ser un instrumento de desarrollo libre, siendo la opción más ajustada a los conocimientos y posibilidades de acceso del equipo de desarrollo. Mediante el uso de metodologías ágiles se buscó asegurar una integración continua y una correcta documentación teniendo en cuenta diagramas y documentos que facilitaron el entendimiento de la estructura de ambas aplicaciones.

El sistema de información web cuenta con diversos niveles de privilegios, de modo que un usuario puede realizar acciones sobre su cultivo o en su defecto, un administrador o súper usuario puede realizar por el usuario, diferentes acciones que el cultivo requiera. Por otro lado, el sistema ofrece la gestión de actividades de modo que el usuario pueda programarlas y estar al tanto de ellas. También, brinda estadísticas del cultivo de acuerdo a las situaciones climáticas que se estén presentando actualmente en la zona donde está presente la plantación.

\section{Funcionamiento de la aplicación móvil}

El sistema está compuesto por un menú de opciones donde se engloban diferentes variables para determinar el estado del suelo. A continuación se presenta una imagen donde se observan las diferentes opciones que presenta el sistema.

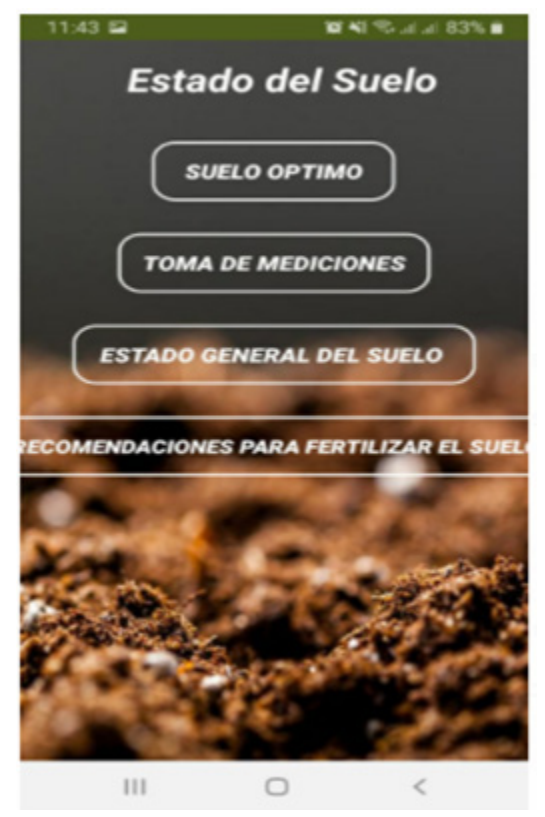

Figura 2. Funcionamiento del sistema

Fuente: Grupo Investigación

\section{1.- Suelo óptimo}

Esta opción indica las características físicas, químicas y biológicas del suelo como lo son: temperatura, humedad, $\mathrm{PH}$, potasio $(K)$, fosforo $(P)$ y nitrógeno $(N)$. A continuación se presenta una imagen donde se observan las opciones de este menú

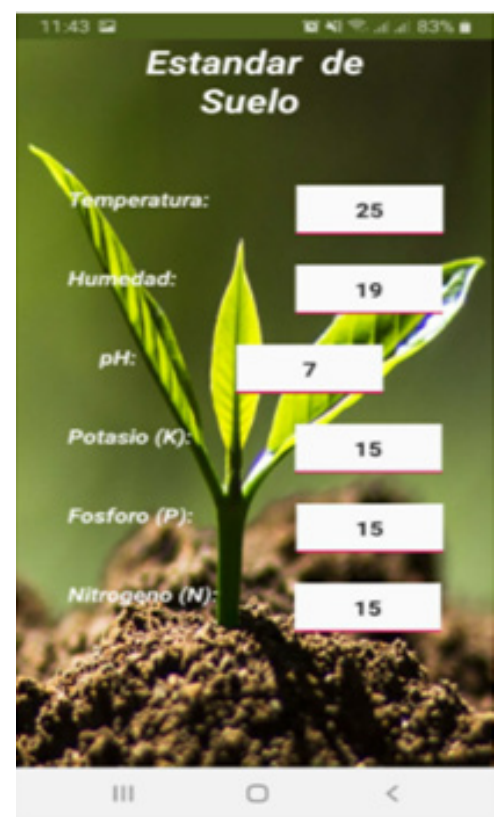

Figura 3. Suelo Óptimo

Fuente: Grupo Investigación 


\section{2.- Toma de Mediciones}

Ésta opción muestra los dispositivos vinculados a la aplicación. A continuación se presenta una imagen donde se observa esta opción.

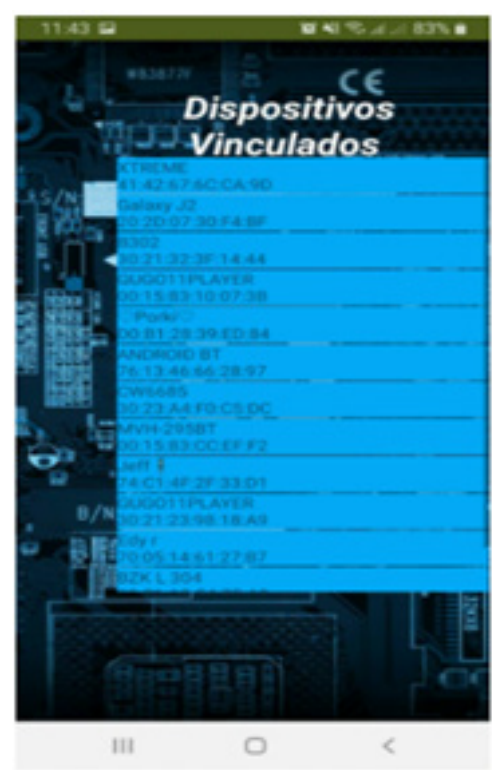

Figura 4. Toma de Mediciones

Fuente: Grupo Investigación

\section{3.- Estado general del suelo}

A través de esta opción se determina el estado del suelo y se evalúan las características físicas, químicas y biológicas que se han estudiado, permitiendo realizar un diagnóstico de acuerdo a los datos recogidos durante el análisis hecho por parte de la aplicación móvil. A continuación se presenta una imagen donde se observa esta opción del menú.

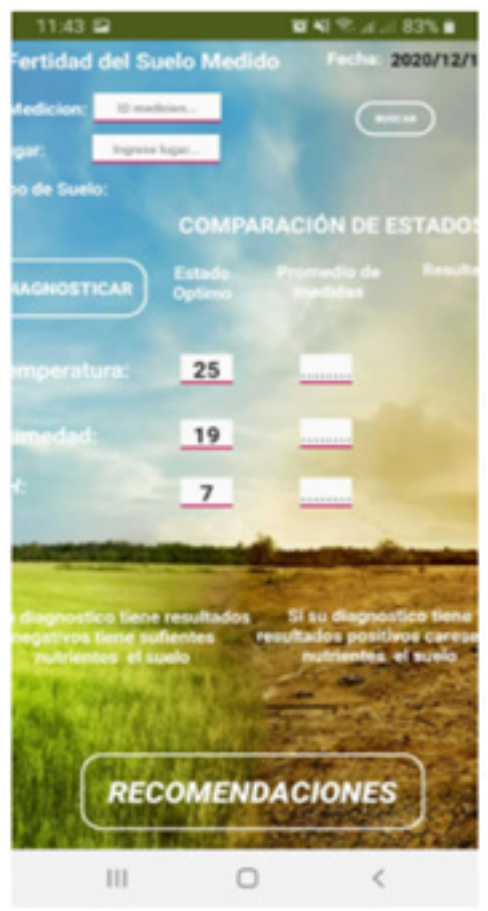

Figura 5. Estado General del Suelo

Fuente: Grupo Investigación

\section{4.- Recomendaciones para fertilizar el suelo}

A través de esta opción la aplicación brinda las recomendaciones necesarias de acuerdo a las características evaluadas dando opciones a los encargados de los suelos para nivelar las variables fisicoquímicas y así, ofrecer un suelo con mejores beneficios para el cultivo. 


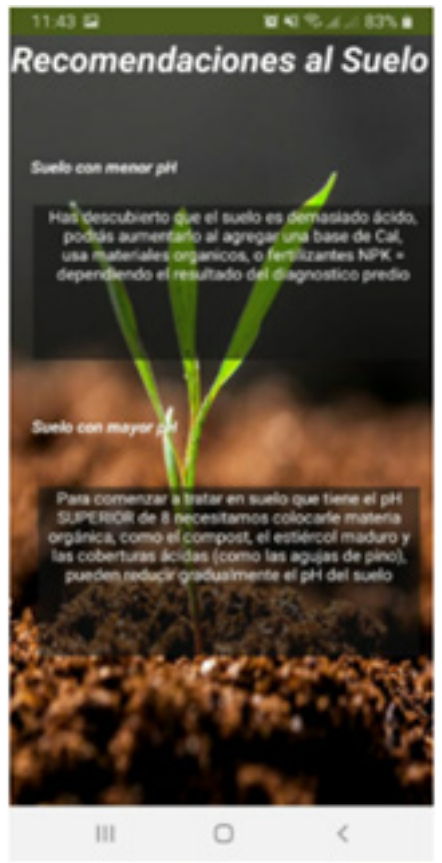

Figura 6. Recomendaciones

Fuente: Grupo Investigación

\section{Resultados}

\section{El sector agrícola}

En el sector agrícola del Ecuador los productores no tienen la capacidad de negociar con los compradores, ya que, los precios de ventas de su producto son establecidos de acuerdo a un listado de referencia. Esto genera varias barreras en los agricultores y en la comercialización de sus productos puesto que las inversiones iniciales son muy elevadas, adicionalmente, los cambios y fenómenos climáticos y el tiempo entre siembra y cosecha son factores que demandan una alta inversión entre las personas que se dedican a esta actividad.

En el caso de las pequeñas y medianas empresas los autores Colloredo, Ordoñez, \& Paltán, (2016) expresan que "se complica aún más el panorama, porque no poseen canales de distribución propios y su poder de negociación es casi nulo con los pocos compradores del sector, es decir con empresas agroindustriales, exportadores y comercializadores nacionales". En tal sentido, al tener pocos compradores, las actividades agrícolas en Ecuador muestran estructuras de oligopsonio y también se aprecia una competencia monopolística a la vez.

\section{Gestión del suelo}

El manejo del suelo es una parte integral de las ocupaciones de la agricultura. Un conocimiento sólido de varios tipos de suelo y sus condiciones, mejoran el rendimiento de los cultivos y conservan los recursos del suelo, a través del uso de operaciones, prácticas y tratamientos para mejorar comportamiento de los mismos. La aplicación de abono y estiércol mejora la porosidad y agregación. El aprovechamiento de estos residuos orgánicos "cobra cada día mayor interés como medio eficiente de reciclaje de nutrimentos, que ayuda al crecimiento de las plantas y devuelven al suelo muchos de los elementos extraídos durante el proceso productivo" (Ramos \& Terry, 2015).

La aplicación de los materiales orgánicos son esenciales para mejorar la calidad del suelo. La producción de hortalizas y otros cultivos comestibles suelen ser significativamente afectados por varios patógenos del suelo que requieren control. El autor (Stivers, 2017) expresa que:

El manejo de la fertilidad del suelo es el análisis de suelos. Un análisis de suelos le provee una información muy importante sobre los niveles de los nutrientes del suelo, incluyendo el fósforo, el potasio, el calcio y el magnesio como también el pH o la acidez del mismo. Usted también puede analizar la materia orgánica. La mayoría de los análisis de suelos no incluyen el nitrógeno. El nitrógeno, es un nutriente muy importante y uno que frecuentemente está deficiente en el suelo (p. 9). 


\section{Rangos de temperatura, humedad y ph}

El análisis de los rangos de temperatura, humedad y ph juega un papel clave en el manejo de la agricultura, ya que, esto asegura el bienestar de la planta, por lo que se debe considerar necesario una medición precisa de estas características del suelo. Hoy en día se emplean sensores que ayudan con la recolección de los datos aportando beneficios en el manejo de los rangos requeridos.

Para poder recoger este tipo de datos en el campo de la agricultura y de acuerdo al manejo de la IA se emplean sensores y arduinos. Los arduinos "son una plaforma de hardware libre, basada en una placa con un micro controlador y un entorno de desarrollo, diseñada para facilitar el uso de la electrónica en proyectos multidisciplinares"(Chora, Álvarez, \& Espinoza, 2018).

De acuerdo con el uso de este tipo de plataformas, los estudios de los suelos son más eficientes y ofrecen mayor calidad del producto, brindando al agricultor un mejor uso de los tiempos de empleo, minimizando los riesgos y las perdidas. En tal sentido, los rangos requeridos para el manejo de la temperatura, el ph y la humedad se presentan a continuación:

\section{Temperatura}

Tabla 1.

\begin{tabular}{|c|c|c|}
\hline Temperatura & Mínima & Máxima \\
\hline Rango & 17 & 23 \\
\hline
\end{tabular}

Fuente: Grupo Investigación
Tabla 2.

\begin{tabular}{|c|c|c|}
\hline Fecha & $\begin{array}{c}\text { Dato } \\
\text { Temperatura }\end{array}$ & $\begin{array}{c}\text { Rango } \\
\text { Optimo }\end{array}$ \\
\hline $2019-12-25$ & 0 & $X$ \\
\hline $2019-12-26$ & 6 & $X$ \\
\hline $2019-12-27$ & 2 & $X$ \\
\hline $2020-01-10$ & 8 & $X$ \\
\hline $2020-01-30$ & 23 & $\checkmark$ \\
\hline $2020-01-31$ & 29.1 & $X$ \\
\hline
\end{tabular}

Fuente: Grupo Investigación

En un estudio realizado en invernaderos entre los meses diciembre del 2019 y enero del 2020 la temperatura fue incrementada con el paso de los días logrando un rango óptimo casi al mes de iniciado los análisis.

\section{Humedad}

Tabla 3.

\begin{tabular}{|c|c|c|}
\hline Humedad & Mínima & Máxima \\
\hline Rango & 5 & 50 \\
\hline
\end{tabular}

Fuente: Grupo Investigación

Tabla 4.

\begin{tabular}{|c|c|c|}
\hline Fecha & $\begin{array}{c}\text { Dato } \\
\text { Temperatura }\end{array}$ & $\begin{array}{c}\text { Rango } \\
\text { Optimo }\end{array}$ \\
\hline $2019-12-25$ & 5 & $\checkmark$ \\
\hline $2019-12-26$ & 0 & $X$ \\
\hline $2019-12-27$ & 4 & $X$ \\
\hline $2020-01-10$ & 6 & $\checkmark$ \\
\hline $2020-01-30$ & 100 & $X$ \\
\hline $2020-01-31$ & 50 & $\checkmark$ \\
\hline
\end{tabular}

Fuente: Grupo Investigación

En el mismo estudio la humedad alcanzó rangos óptimos en tres oportunidades presentando un ascenso significativo el día 30/01 el cual fue controlado para el día siguiente. 
PH

Tabla 5.

\begin{tabular}{|c|c|c|}
\hline PH & Mínima & Máxima \\
\hline Rango & 7 & 7 \\
\hline
\end{tabular}

Fuente: Grupo Investigación

Tabla 6.

\begin{tabular}{|c|c|c|}
\hline Fecha & $\begin{array}{c}\text { Dato } \\
\text { Temperatura }\end{array}$ & $\begin{array}{c}\text { Rango } \\
\text { Optimo }\end{array}$ \\
\hline 2019-12-25 & 7 & $\checkmark$ \\
\hline $2019-12-26$ & 7 & $\checkmark$ \\
\hline $2019-12-27$ & 7 & $\checkmark$ \\
\hline $2020-01-10$ & 7 & $\checkmark$ \\
\hline 2020-01-30 & 7 & $\checkmark$ \\
\hline 2020-01-31 & 7 & $\checkmark$ \\
\hline
\end{tabular}

Fuente: Grupo Investigación

En el estudio a los trabajadores florícolas se les realizó una encuesta con diferentes preguntas dando a conocer los siguientes resultados:

\section{ANEXOS}

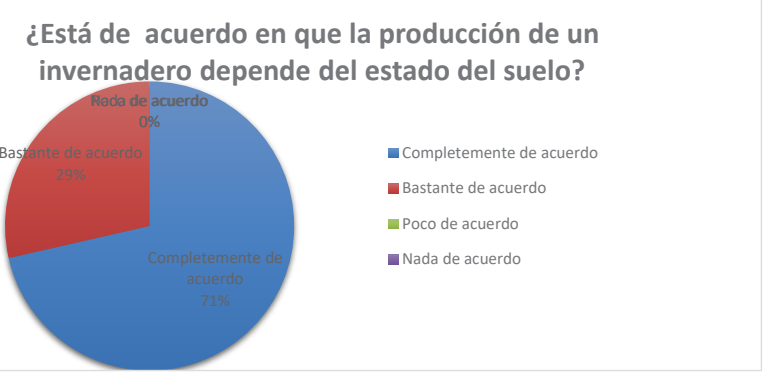

Figura 7. ¿Está de acuerdo en que la producción de un invernadero depende del estado del suelo?

Fuente: Grupo Investigación

Análisis: Con la información obtenida de la siguiente encueta, un $71 \%$ de trabajadores de Floricolas manifiestan que la produc- ción de un invernadero depende del estado del suelo, un $29 \%$ está bastante de acuerdo, por lo que se puede decir que la mayoría de los trabajadores dice que la producción si depende del estado del suelo.

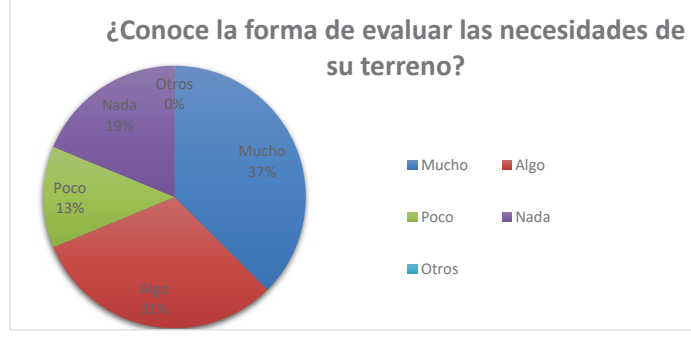

Figura 8. ¿Conoce la forma de evaluar las necesidades de su terreno?

Fuente: Grupo Investigación

Análisis: La encuesta realizada brinda la siguiente información, un 37\% de trabajadores de Florícolas manifiestan si conocen mucho la forma de evaluar al terreno, un $31 \%$ comentan que conoce algo no más de como evaluar al terreno para la producción, un $19 \%$ dicen que no conocen nada del tema, un $13 \%$ comenta que conocen muy poco del tema de evaluar las necesidades del terreno, como conclusión se puede evaluar que el porcentaje mayor es un 37\% que conocen la forma de evaluar las necesidades del terreno para la producción.

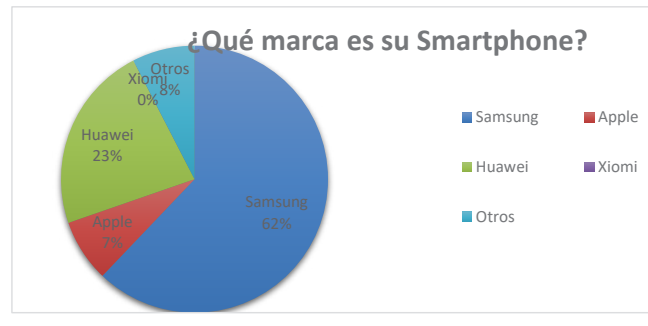

Figura 9. ¿Qué marca es su Smartphone?

Fuente: Grupo Investigación

Análisis: Según los resultados obtenidos un $62 \%$ de trabajadores de Floricolas poseen un Smartphone de marca Samsung, un 23\% manifiesta que tiene un Smartphone de la marca huawei, un $7 \%$ de trabajadores dicen que poseen un teléfono Apple, 
llegando a la conclusión de que la gran mayoría que es un $62 \%$ de trabajadores tienen un teléfono Samsung.

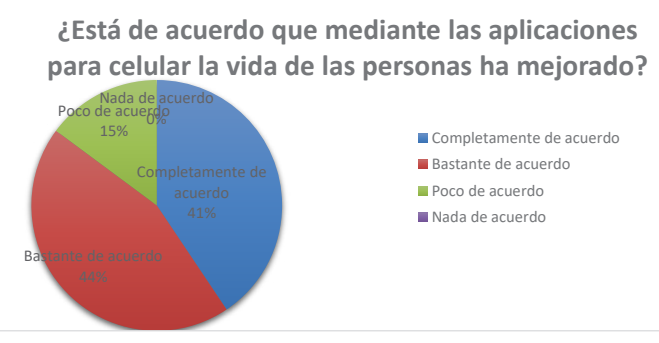

Figura 10. ¿Está de acuerdo que mediante las aplicaciones para celular la vida de las personas ha mejorado?

Fuente: Grupo Investigación

Análisis: Se observa que un $44 \%$ de los trabajadores de Florícolas se encuentra bastante de acuerdo que mediante aplicaciones del teléfono la vida de las personas ha mejorado, un $41 \%$ está de completamente de acuerdo a que un celular puede mejorar la vida de las personas, un 15\% dice que no se encuentra ni de acuerdo ni en desacuerdo con el tema, como conclusión la mayoría de trabajadores creen que una aplicación del teléfono mejora su vida.

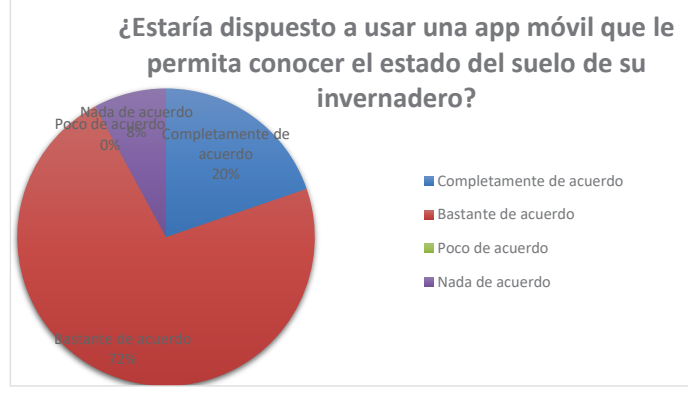

Figura 11. ¿Estaría dispuesto a usar una app móvil que le permita conocer el estado del suelo de su invernadero?

Fuente: Grupo Investigación

Análisis: El 72\% está bastante de acuerdo en usar una app móvil que le permita conocer el estado del suelo de su invernadero, un $20 \%$ manifiesta que si está completa- mente de acuerdo usar una app móvil que le permita conocer el estado del suelo de su invernadero, un 0\% no están de acuerdo ni en desacuerdo, como conclusión los trabajadores de las agrícolas se encuentran de acuerdo el utilizar el teléfono para diferentes usos.

Durante el mismo estudio se realizaron una serie de preguntas a los ingenieros en sistemas encargados de la producción del invernadero. A continuación se presentan las preguntas y análisis.

¿Conoce usted sobre la producción de un invernadero optimizado mediante un arduino?

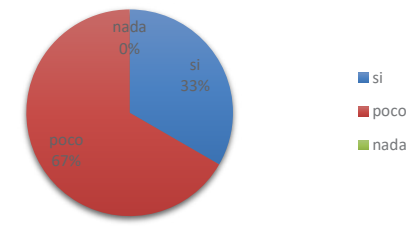

Figura 12. ¿Conoce usted sobre la producción de un invernadero optimizado mediante un arduino?

Fuente: Grupo Investigación

Análisis: Con la encuesta realizada a los Ingenieros en Sistemas se obtiene los siguientes resultados, un $67 \%$ de Ingeniero conoce poco sobre la producción de un invernadero optimizado mediante un Arduino, un $33 \%$ manifiesto que conoce muy bien sobre la producción de un invernadero optimizado mediante un Arduino y un 0\% comentan no conocer sobre el tema, llegando a la conclusión que poco se conoce de la producción de un invernadero optimizado mediante un Arduino. 
¿Tiene experiencia con una placa que admite sensores que recolectan información?

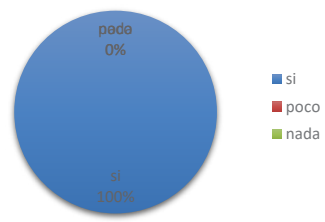

Figura 13. ¿Tiene experiencia con una placa que admite sensores que recolectan información?

Fuente: Grupo Investigación

Análisis: Con la información obtenida en la encueta, un $100 \%$ dice tener experiencia con una placa que admite sensores que recolectan información.

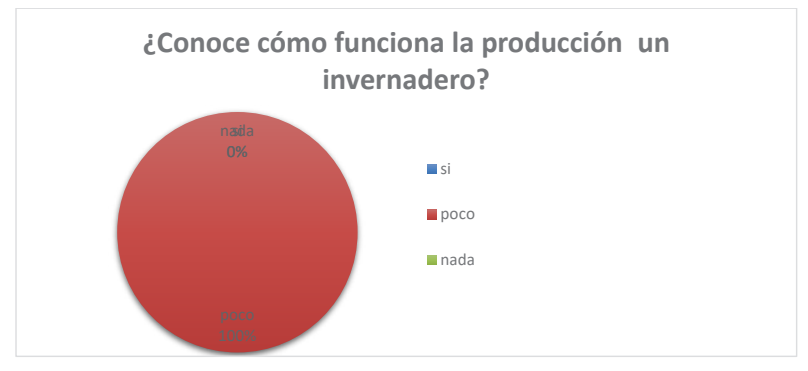

Figura 14. ¿Conoce cómo funciona la producción un invernadero?

Fuente: Grupo Investigación

Análisis: El 100\% conoce cómo funciona la producción de un invernadero.

Considera usted que el uso de software
sea importante en un invernadero?

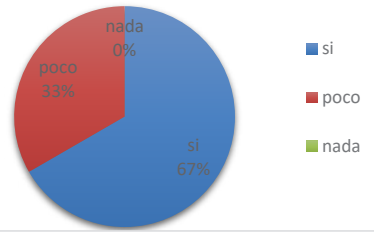

Figura 15. ¿Considera usted que el uso de software sea importante en un invernadero?

Fuente: Grupo Investigación
Análisis: El 67\% de los Ingenieros en sistemas manifiestan que el uso de software es importante en un invernadero, un 33\% cree que no se necesita mucho un software, por lo tanto se concluye que la mayoría de los Ingeniero si creen que es importante el uso de un software en los invernadero.

¿Cree usted que es bueno implementar un proyecto sistemático en un invernadero?

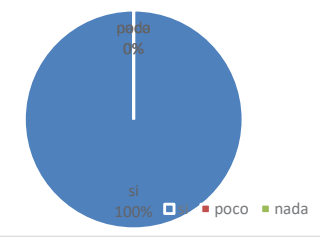

Figura 16. ¿Cree usted que es bueno implementar un proyecto sistemático en un invernadero?

Fuente: Grupo Investigación

Análisis: Se observa que el 100\% cree que es bueno implementar un proyecto sistemático en un invernadero es decir la mayoría está de acuerdo.

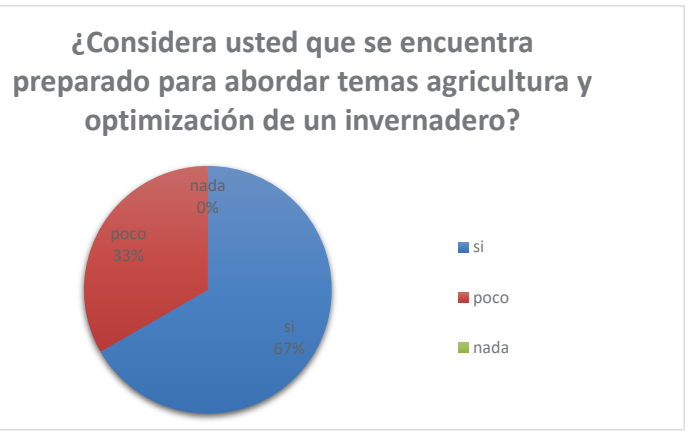

Figura 17. ¿Considera usted que se encuentra preparado para abordar temas agricultura y optimización de un invernadero?

Fuente: Grupo Investigación

Análisis: El 67\% manifiestan que se encuentran preparados para abordar, un 33\% comentan que está que no se encuentran preparados para impartir el tema, por lo que se concluye que debe existir un adiestra- 
miento previo a los ingenieros encargados de los sistemas para poder abordar temas de optimización de invernaderos.

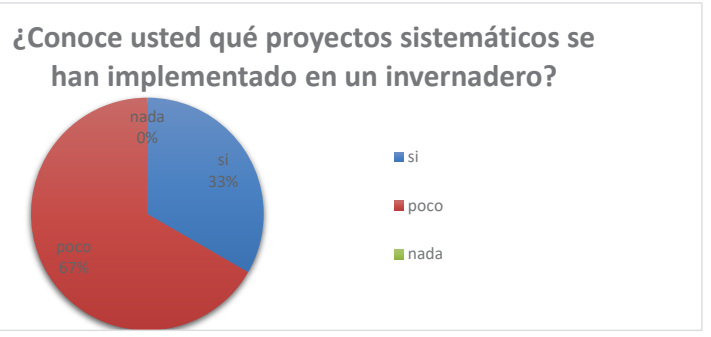

Figura 18. ¿Conoce usted qué proyectos sistemáticos se han implementado en un invernadero?

Fuente: Grupo Investigación

Análisis: El $33 \%$ de Ingenieros en Sistemas conoce proyectos sistemáticos que se han implementado en un invernadero, un $67 \%$ conoce muy poco sobre proyectos sistemáticos, mientras que el $0 \%$ no tienen nada de conocimientos de cómo manejar proyectos sistemáticos. Se concluye que existe un alto porcentaje con este tipo de conocimientos y se observa la necesidad de emplear estudios referentes al tema.

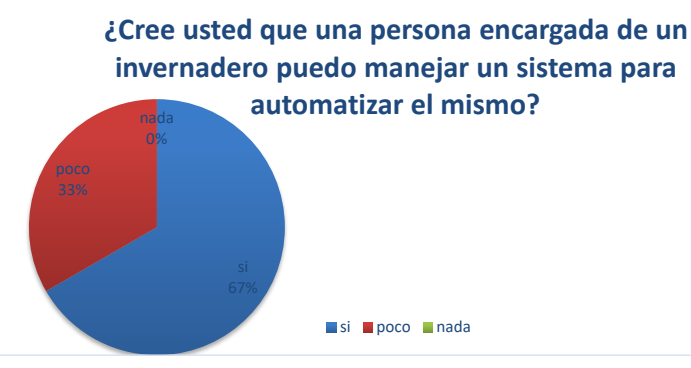

Figura 19. - ¿Cree usted que una persona encargada de un invernadero puedo manejar un sistema para automatizar el mismo?

Fuente: Grupo Investigación

Análisis: El 67\% de Ingenieros cree que una persona encargada de un invernadero puedo manejar un sistema para automatizar el mismo, mientras que un 33\% conoce muy poco de cómo manejar un sistema para automatizar, por tanto, se observa que un alto porcentaje de Ingenieros si tiene conocimiento del tema.

\section{Control de malezas dentro de invernade- ros y estructuras cerradas}

Las malezas de cultivos en contenedores en estructuras cerradas son generalmente más problemáticas en el vivero que en los cultivos de invernadero, principalmente porque la mayoría de los cultivos de invernadero se cultivan con medios de mayor calidad relativamente libre de semillas de y malezas. Sin embargo, las malezas siguen causando problemas en los cultivos de floricultura debido a las prácticas culturales deficientes. "El mantillo de grava y tela puede reducir la germinación de malezas, pero en algunos casos, la grava se llenará con sustrato para macetas y restos de cultivos, negando sus beneficios" (Pernilla, 2016)

Las malezas germinan fácilmente a lo largo de los bordes y en las rasgaduras y áreas desgastadas de la tela de bloques de malezas. La exploración es fundamental para determinar si las semillas de malezas están germinando en macetas. "Maleza debajo de bancos oen los pasillos puede que no afecten directamente el crecimiento de las plantas, pero albergan plagas (pulgones, moscas blancas, trips y ácaros). El control de malezas más rentable es la prevención y el uso de químicos y métodos no químicos para un programa de manejo de plagas verdaderamente integrado (MIP).

\section{Saneamiento y Prevención}

El saneamiento y la prevención son los primeros pasos fundamentales en todos los programas de MIP. "El medio ambiente del invernadero, reduce la cantidad de semillas de malezas que ingresan, pero las semillas de malezas aún se pueden volaren invernaderos o ser transportados por agua" (PerniIla, 2016). 
Los pisos de concreto o las telas de barrera contra las malezas colocadas sobre la grava ayudarán a prevenir la germinación de las malezas y son mucho más fáciles de mantener libres de malezas que el suelo o la grava muy contaminada. Lavar contenedores para su reutilización con agua a presión y desinfectantes químicos para eliminar la suciedad, los patógenos y semillas de malezas

\section{Deshierbe manual}

Algunas de las malezas de invernadero más comunes incluyen spurge, bittercress y oxalis. "Estas malas hierbas producen miles de semillas que germinan rápidamente. Exploración continua y la eliminación es fundamental para detener la floración y la producción de semillas" (Pernilla, 2016). Dejando malezas en el suelo del invernadero permitirá que algunos de ellos vuelvan a enraizar y las semillas que quedan en estas malezas aún pueden germinar y contaminar el invernadero con una nueva cosecha de plántulas. No es infrecuente que las malas hierbas arrancadas para continuar floreciendo y produciendo nuevas semillas en condiciones benignas de invernadero.

\section{Prácticas de control cultural}

Las algas, el musgo y las hepáticas prosperan en ambientes húmedos que son comunes en los invernaderos. Desafortunadamente, el riego excesivo de cultivos de invernadero también es común y conduce a muchos problemas, incluidas las enfermedades de los cultivos y las condiciones ideales para el crecimiento de estos plagas verdes.

Pueden cubrir rápidamente superficies de medios para macetas, concreto, grava y tierra. pisos de tela así como superficies de bancos. Se convierten en un grave problema para la seguridad de los trabajadores cuando cubren la superficie del piso haciendo que los pisos sean resbalosos.(Pernilla, 2016)

\section{Métodos de control químico}

Hay muy pocos herbicidas etiquetados para su uso dentro de invernaderos debido al potencial de cultivo daño por volatilización. Es mejor utilizar métodos no químicos cuando sea posible, pero los herbicidas se puede utilizar para controlar las malas hierbas que se escapan.

La mayoría de los herbicidas etiquetados para uso en invernaderos son herbicidas postemergentes, que controlan malezas después de que germinan. Es importante tener en cuenta que se necesita una cobertura completa para obtener control adecuado con herbicidas de contacto y todos los herbicidas post emergentes funcionan mejor cuando las malezas son pequeños y están creciendo activamente.

\section{Desafíos de la IA en la agricultura}

Los sistemas expertos son herramientas para la gestión agrícola y pueden proporcionar sitios específicos, integrados e interpretados. Sin embargo, el desarrollo de sistemas expertos para la agricultura es bastante reciente, y el uso de estos sistemas en la agricultura comercial es raro hasta la fecha.

Aunque la IA hizo una mejora notable en el sector agrícola, todavía tiene un impacto por debajo del promedio en las actividades de la agricultura en comparación con sus potenciales e impactos en otros sectores. Sin embargo, aún queda mucho por hacer para mejorar la agricultura e incorporar actividades que utilizan IA, ya que existen muchas limitaciones para su implementación entre las que se mencionan:

\section{A. Limitación: tiempo de respuesta y pre- cisión}

Un atributo importante de un sistema inteligente o experto es su capacidad para ejecutar tareas con precisión en muy poco 
tiempo. La mayoría de los sistemas se quedan cortos en tiempo de respuesta o precisión, o incluso ambos. Un retraso del sistema afecta la selección de estrategias y tareas. Se hipotetiza que la selección de estrategia se basa en una función de costos que combina dos factores: (1) el esfuerzo requerido para sincronizar la disponibilidad del sistema de entrada y (2) la precisión nivel permitido. "Las personas que buscan minimizar el esfuerzo y maximizar precisión, deben elegir entre tres estrategias: automática rendimiento, estimulación y seguimiento"(Duckett \& Pearson, 2018).

\section{B. Limitación 2: Big Data Requerido}

La fuerza de un agente inteligente también se mide en el volumen de datos de entrada. Un sistema de IA en tiempo real necesita monitorear un inmenso volumen de datos. "El sistema debe filtrar mucho de los datos entrantes. Sin embargo, debe seguir respondiendo a acontecimientos importantes o inesperados" (Duckett \& Pearson, 2018). En este caso, se requiere de un experto que tenga el conocimiento necesario para manejar el sistema de campo a través de datos relevantes que mejoren la velocidad y la precisión.

\section{Limitación 3: Método de implementa- ción}

La eficacia de cualquier sistema experto radica en su ejecución y la metodología. Dado que utiliza big data, el método de búsqueda y el entrenamiento debe definirse adecuadamente en cuanto a velocidad y precisión.

\section{Limitación 4: Alto costo de datos}

La mayoría de los sistemas de inteligencia artificial están basados en Internet, lo que a su vez reduce y restringe su uso, particularmente en áreas remotas o rurales. "El gobierno puede ayudar a los agricultores diseñando una web dispositivo habilitador de servicio con tarifa más baja para trabajar de forma exclusiva los sistemas de IA para los agricultores" (Duckett \& Pearson, 2018). Además, una forma de "cómo usar "la orientación (capacitación y reentrenamiento) realmente ayudará a los agricultores adaptarse al uso de la IA en la granja.

\section{E. Limitación 5: Flexibilidad}

La flexibilidad es un atributo importante de cualquier sistema de IA de sonido. "Se percibió que se ha avanzado mucho en la aplicación de la IA por medio de técnicas para tareas particularmente aisladas" (Duckett \& Pearson, 2018), a pesar de que el tema importante a la vanguardia de la tecnología robótica basada en IA parece para ser la interfaz de los subsistemas en un integrado del medio ambiente.

\section{Conclusiones}

Es evidente que la industria agrícola enfrenta varios desafíos, como la falta de sistemas de riegos efectivos, malezas, problemas con el monitoreo de plantas debido al manejo adecuado en los diferentes campos tanto abierto como cerrado para el tratado de plantas y cultivos. Sin embargo y durante la evaluación literaria se observó que el rendimiento se puede aumentar con la ayuda de la tecnología logrando minimizar este tipo de problemas.

Las diferentes técnicas impulsadas por la IA como sensores remotos para la detección del contenido de humedad, la temperatura y el $\mathrm{PH}$ del suelo, así como también, el riego automatizado con la ayuda de GPS brindan mejoras en el manejo de técnicas aplicadas en la agricultura. No obstante, la aplicación de sistemas avanzados de IA presenta limitantes que van ligados al manejo e implementación de los equipo y al conocimiento de los mismos por parte del personal que labora en el sector agrícola.

Tal y como se observó el personal floricultora muy a pesar de poseer teléfonos inte-

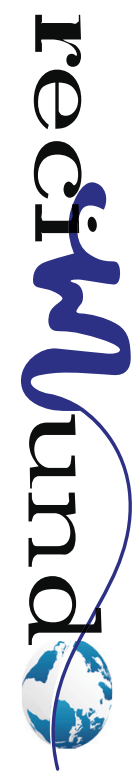


ligentes y estar a la disposición del manejo del suelo a través de apps móviles, muchos de ellos, no tenía idea de cómo manejar la información digital y aprovecharla en todo sentido, aunado a ello no todos los ingenieros de sistemas conocen del manejo de los Arduinos y las placas para el control por lo que se observa la necesidad de un entrenamiento por parte de los dueños de fincas e invernaderos con el fin de conocer el uso de tecnologías que brindan mejorías a los suelos y que logran predecir situaciones a futuro.

La escasez de recursos y puestos de trabajo puede entenderse con la ayuda del poder intelectual creado por el hombre en cuestiones de agronegocios. En las estrategias convencionales se requería una gran cantidad de trabajo para obtener características del cultivo como altura de la planta, textura y contenido del suelo, de esta manera se realizaban pruebas manuales que resultaban tediosas. Por tal razón, con el empleo de las IA se perciben grandes mejoras en varios sistemas examinados y se produce un alto y rápido rendimiento no dañino que ofrece mayores ventajas en el sector agrícola tanto en campo abierto como cerrado y un acceso más controlado a la información.

\section{Bibliografía}

Bolívar, J. (2015). Investigación Documental. México. Pax.

Castro, J. (2016). Técnicas Documentales. México. Limusa.

Chora, D., Álvarez, S., \& Espinoza, M. (2018). Raspberry Pi y Arduino: semilleros en innovación tecnológica para la agricultura de precisión. Revista de tecnologias de la informatica y las telecomunicaciones, Vol. 2 (1), 74-82.

Davila, A. (2015). Diccionario de Términos Científicos. .Caracas: Editorial Oasis.

Duckett, S., \& Pearson, S. (2018). Agricultural Robotics: Robotics: The Future of Robotic Agriculture. UK-RAS.

Liakos, K., \& Busato, P. (2018). Machine Learning in
Agriculture: A Review. Sensors. 18, 1-29.

Ortiz, P., \& Gutiérrez, J. P. (2016). "Paradigmas de aprendizaje automático para el mapeo de malezas a través de malezas a través de Vehículos aéreos no tripulados ",. Serie de simposios sobre inteligencia computacional, Atenas, Grecia.

Pernilla, B. (2016). Where, when and how plant-soil feedback matters in a changing world. Funct. Ecol. , 30, 1109-1121.

Ramos, D., \& Terry, A. (2015). Generalidades de los abonos orgánicos: Importancia del Bocashi como alternativa nutricional para suelos y plantas. Cultivos Tropicales , 35 (4).

Snehal, S., \& Sandeep, S. (2014). Agricultural crop yield prediction using Research in Electrical, Electronics. Instrumentation and Control Engineering, 2 (1), 683-686.

SofOs. (2017, Diciembre 8). Impacto de la tecnología aplicada en la agricultura. Retrieved Diciembre 29, 2020, from http://www.sofoscorp.com/impacto-tecnologia-aplicada-agricultura/

Stivers, L. (2017, Octubre). Introducción a los Suelos: El Manejo de los Suelos. Retrieved Diciembre 29, 2020, from https://extension.psu.edu/introduccion-a-los-suelos-el-manejo-de-los-suelos

Zibah, G. (2015). Agricultural production and economic growth: Implication for rural poverty alleviation". Quarterly Journal of International Agriculture, 53 (3), 207-223. 


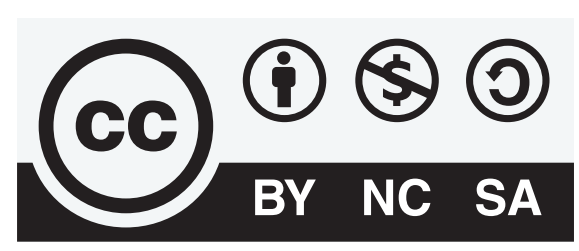

CREATIVE COMMONS RECONOCIMIENTO-NOCOMERCIAL-COMPARTIRIGUAL 4.0.

\section{CITAR ESTE ARTICULO:}

Bonilla Segovia, J. S., Dávila Rojas, F. A., \& Villa Quishpe, M. W. (2021). Estudio del uso de técnicas de inteligencia artificial aplicadas para análisis de suelos para el sector agrícola. RECIMUNDO, 5(1), 4-19. https://doi.org/10.26820/recimundo/5.(1).enero.2021.4-19 